\title{
BIFURCATION AND STABILITY ANALYSIS \\ OF A ROTATING BEAM
}

\author{
BY \\ PETER GROSS (Mathematical Institute, The Technical University of Denmark, Copenhagen) \\ METIN GÜRGÖZE (Safety Control Engineering, University of Wuppertal, Wuppertal, Germany) \\ AND \\ WOLFHARD KLIEM (Mathematical Institute, The Technical University of Denmark, Copenhagen)
}

\begin{abstract}
We discuss small oscillations of an elastic beam clamped radially to the interior of a rotating ring. It is well known that if the speed of rotation is sufficiently high, the trivial equilibrium of the beam may lose stability and the beam buckles.

Earlier papers on this subject have, with a few exceptions, dealt with linear models and thus only considered the trivial equilibrium of the beam. In the present work, a nonlinear model including third-order terms is proposed. Using a Ritz-Galerkin procedure, the model equation is reduced to a nonlinear ordinary differential equation (ODE) of second order, which contains no Coriolis terms. Previous nonlinear models in the literature have Coriolis terms as the governing nonlinear effects, but this has been shown to be erroneous.

This nonlinear ODE is investigated by phase plane techniques. Bifurcation and stability analysis reveals three different types of behaviour, depending on the length of the beam relative to the ring radius. Bifurcation diagrams and associated phase portraits are given.

The theoretical basis for the applied phase plane techniques is the fact that the reduced equation is conservative, i.e., possesses a first integral. An appendix briefly outlines the most important consequences of this property.
\end{abstract}

1. Introduction. Stability problems of elastic structures attached to the interior of a rotating wheel have been investigated for many years, since rotating wheels with clamped beams and plates exist in machinery like centrifuges and turbines, in wobbling rotors and in several other physical systems. Many papers have concentrated on buckling of rotating rods, e.g., Mostaghel and Tadjbakhsh [1], Rammerstorfer [2], Nachman [3], and Wang [4]. In addition to this, the dynamical behaviour of rotating rods has also been investigated, e.g., by Lakin and Nachman [5], Peters and Hodges [6], and Gürgöze [7].

Received July 2, 1991.

1991 Mathematics Subject Classification. Primary 73H05, 70K05.

(C)1993 Brown University 
All these papers deal with linear models only, and thus only the stability of the trivial equilibrium may be investigated. To investigate the occurrence and stability of other (buckled) equilibria, a nonlinear model is required. In the literature, however, only a few nonlinear models are proposed, e.g., Weber [8] and Hernried and Gustafson [9]. These two papers suffer the weakness that a Coriolis term of second order is considered an essential feature in the nonlinear differential equation, whereas the presence of such a term was shown to be erroneous by Peters and Hodges [10] and Kliem [11].

The present work establishes an integrodifferential equation as a nonlinear model of a vibrating elastic beam clamped radially to the interior of a steadily rotating rigid ring. Using a simple polynomial of fourth degree within a Galerkin procedure, this nonlinear model leads to a nonlinear second-order autonomous ordinary differential equation (ODE), which becomes the subject of a bifurcation and stability analysis. The results due to the linear terms of the model are compared with those of earlier investigations.

Finally, a few additional methods suitable for an analysis of the problem are briefly discussed.

2. The nonlinear model. Referring to Fig. 1, we consider an elastic beam or rod with length $L$, constant mass per unit length $\mu$, and bending stiffness $E I$. The beam is clamped radially to the interior of a rigid ring with radius $R$. The ring rotates with constant angular velocity $\Omega$ about an axis through the center of the ring and perpendicular to the plane of the ring. Small vibrations of the beam are assumed to exist only in this plane.

Denote by $\sigma$ the length along the beam measured from the clamped end, by $t$ the time, and by $v(\sigma, t)$ the lateral deflection of beam elements.

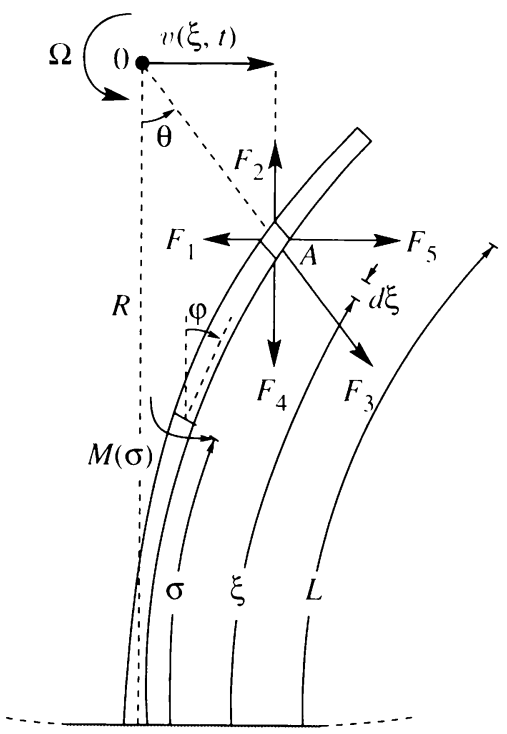

FIG. 1. Elastic beam clamped to a rotating ring. $F_{1}, \ldots, F_{5}$ are the inertia forces active at every beam element. 
The governing equation for an Euler-Bernoulli beam is

$$
E I \kappa(\sigma)=M(\sigma),
$$

where $\kappa(\sigma)=v_{\sigma \sigma}\left(1-v_{\sigma}^{2}\right)^{-1 / 2}$ is the curvature $\sigma$ with the usual abbreviations $v_{\sigma}=$ $\partial v / \partial \sigma$ and $v_{\sigma \sigma}=\partial^{2} v / \partial \sigma^{2}$. The bending moment $M(\sigma)$ is due to the inertia forces $F_{1}, \ldots, F_{5}$, which are active at every beam element of length $d \xi$ if a reference system fixed to the beam is used (Fig. 1). Notice that the model does not include rotation of beam cross sections, influence of shearing effects, and gravity.

The forces $F_{1}=\mu v_{t t} d \xi$ and $F_{2}=\mu\left(\xi-\int_{0}^{\xi} \cos \varphi d \eta\right)_{t t} d \xi$ are due to the acceleration relative to ring, whereas the centrifugal force $F_{3}=\mu|O A| \Omega^{2} d \xi$ is due to the rotation of the ring. The Coriolis terms $F_{4}=2 \mu \Omega v_{t} d \xi$ and $F_{5}=$ $-2 \mu \Omega\left(\xi-\int_{0}^{\xi} \cos \varphi d \eta\right)_{t} d \xi$ are due to the rotation of the ring and to the relative velocity of the beam element in the rotating reference system. Then Eq. (1) yields immediately

$$
E I v_{\sigma \sigma}\left(1-v_{\sigma}^{2}\right)^{-1 / 2}=\sum_{i=1}^{5} M_{i}
$$

with

$$
\begin{aligned}
& M_{1}=-\int_{\sigma}^{L} \mu v_{t t} \int_{\sigma}^{\xi} \cos \varphi d \eta d \xi \\
& M_{2}=-\int_{\sigma}^{L} \mu\left(\xi-\int_{0}^{\xi} \cos \varphi d \eta\right)_{t t} \int_{\sigma}^{\xi} \sin \varphi d \eta d \xi \\
& M_{3}=\int_{\sigma}^{L} \mu \Omega^{2}|O A| \cos \theta \int_{\sigma}^{\xi} \sin \varphi d \eta d \xi+\int_{\sigma}^{L} \mu \Omega^{2}|O A| \sin \theta \int_{\sigma}^{\xi} \cos \varphi d \eta d \xi, \\
& M_{4}=\int_{\sigma}^{L} 2 \mu \Omega v_{t} \int_{\sigma}^{\xi} \sin \varphi d \eta d \xi \\
& M_{5}=\int_{\sigma}^{L}-2 \mu \Omega\left(\xi-\int_{0}^{\xi} \cos \varphi d \eta\right)_{t} \int_{\sigma}^{\xi} \cos \varphi d \eta d \xi .
\end{aligned}
$$

In order to simplify Eq. (2), we notice that

$$
\left.\begin{array}{l}
\sin \varphi=v_{\eta}, \quad \cos \varphi=\left(1-v_{\eta}^{2}\right)^{1 / 2}, \\
|O A| \cos \theta=R-\int_{0}^{\xi} \cos \varphi d \eta, \quad|O A| \sin \theta=\int_{0}^{\xi} \sin \varphi d \eta .
\end{array}\right\}
$$

Now we substitute (4) into the expressions (3). In order to obtain the simplest nonlinear approximation to Eq. (2), we replace $\left(1-v_{\eta}^{2}\right)^{1 / 2}$ and $\left(1-v_{\sigma}^{2}\right)^{-1 / 2}$ by the Taylor polynomials $\left(1-\frac{1}{2} v_{\eta}^{2}\right)$ and $\left(1+\frac{1}{2} v_{\sigma}^{2}\right)$, respectively. This makes sense since the deflection $v$ and all its partial derivatives are assumed to be small everywhere along the beam. Furthermore, the introduction of nondimensional parameters

$$
s=\sigma / L, \quad f(s, t)=v(s L, t) / L, \quad \alpha=R / L, \quad \beta=\mu L^{4} / E I
$$


is helpful. Then Eq. (2), by simple but lengthy computations, implies the partial integrodifferential equation

$$
\begin{aligned}
& f_{s s s s}+\frac{1}{2} f_{s s s s} f_{s}^{2}+3 f_{s} f_{s s} f_{s s s}+f_{s s}^{3} \\
& +\beta\left\{f_{s} f_{s s} \int_{s}^{1} f_{t t}(\xi, t) d \xi+f_{t t}-\frac{1}{2} f_{t t} f_{s}^{2}\right. \\
& \quad-f_{s s} \int_{s}^{1} \int_{0}^{\xi}\left(f_{s t}(\eta, t)^{2}+f_{s}(\eta, t) f_{s t t}(\eta, t)\right) d \eta d \xi \\
& \left.\quad+f_{s} \int_{0}^{s}\left(f_{s t}(\eta, t)^{2}+f_{s}(\eta, t) f_{s t t}(\eta, t)\right) d \eta\right\} \\
& +\beta \Omega^{2}\left\{\alpha(1-s) f_{s s}-\frac{1}{2}\left(1-s^{2}\right) f_{s s}+\frac{1}{2} f_{s s} \int_{s}^{1} \int_{0}^{\xi} f_{s}(\eta, t)^{2} d \eta d \xi\right. \\
& \left.\quad-(\alpha-s) f_{s}-\frac{1}{2} f_{s} \int_{0}^{s} f_{s}(\xi, t)^{2} d \xi-f_{s} f_{s s} \int_{0}^{1} f(\xi, t) d \xi-f+\frac{1}{2} f f_{s}^{2}\right\} \\
& 2 \beta \Omega\left\{f_{s s} \int_{s}^{1} f_{t}(\xi, t) d \xi-f_{s} f_{t}+\int_{0}^{s} f_{s}(\eta, t) f_{s t}(\eta, t) d \eta\right\}=0 .
\end{aligned}
$$

The terms containing $\Omega^{2}$ are due to the centrifugal forces, and the terms containing $\Omega$ are due to the Coriolis forces. In the following, it turns out that these Coriolis terms have no influence on the dynamics of the model.

The well-known linear dynamic model, e.g., Gürgöze [7], is formed by the linear terms of Eq. (6):

$$
f_{s s s}+\beta f_{t t}+\beta \Omega^{2}\left[\left\{\alpha(1-s)-\frac{1}{2}\left(1-s^{2}\right)\right\} f_{s s}-(\alpha-s) f_{s}-f\right]=0 .
$$

Finally, the associated linear static model can be established from Eq. (7) by inserting $f_{t t}=0$.

3. Bifurcation and stability analysis. In this section, we analyze Eq. (6) by the method of Galerkin and phase plane analysis. The static linear equation derived from (7) was investigated by Rammerstorfer [2], who dealt with buckling. To determine the critical value of $\beta \Omega^{2}$, he used the Ritz-Galerkin assumption

$$
p(s)=P_{1}(s)=k\left(6 s^{2}-4 s^{3}+s^{4}\right), \quad s \in[0,1], k \neq 0,
$$

which satisfies the boundary conditions

$$
p(0)=p^{\prime}(0)=p^{\prime \prime}(1)=p^{\prime \prime \prime}(1)=0,
$$

corresponding to a clamped end at $s=0$ and a free end at $s=1$.

Similarly, to investigate the dynamics of the rod, we make the Ritz-Galerkin assumption for the first mode of oscillation

$$
f(s, t)=x(t) \cdot\left(6 s^{2}-4 s^{3}+s^{4}\right) .
$$

Substituting Eq. (10) into Eq. (6) and using Galerkin's procedure leads to the nonlinear second-order autonomous ODE

$$
\left(A+B x^{2}\right) \ddot{x}+a x(\dot{x})^{2}+b x+c x^{3}=0,
$$


where

$$
\left.\begin{array}{l}
A=2860 \beta, \quad B=20118 \beta, \\
a=35520 \beta, \quad b=35640+495(1-9 \alpha) \beta \Omega^{2}, \\
c=58320-2358 \beta \Omega^{2} .
\end{array}\right\}
$$

It should be noticed that the calculations involved in the derivation of (11) are rather cumbersome and were carried out in REDUCE, a software package for symbolic calculations, which is described in $[12,13]$.

We notice that no Coriolis terms of the form $\Omega x \dot{x}$ occur in Eq. (11). In general, we may show that the Coriolis terms in Eq. (6) vanish in the Galerkin procedure if we use an arbitrary separation assumption. Indeed, if we assume

$$
f(s, t)=p(s) \cdot x(t),
$$

where $p(s)$ is any function satisfying the boundary conditions (9), the coefficient of $\Omega x \dot{x}$ in the reduced equation is given by

$$
\begin{aligned}
G & =\int_{0}^{1} p(s)\left[p^{\prime \prime}(s) \int_{s}^{1} p(\xi) d \xi-p^{\prime}(s) p(s)+\int_{0}^{s} p^{\prime}(\xi)^{2} d \xi\right] d s \\
& =\int_{0}^{1} p(s)\left[p^{\prime \prime}(s) \int_{s}^{1} p(\xi) d \xi-\int_{0}^{s} p(\xi) p^{\prime \prime}(\xi) d \xi\right] d s \\
& =\int_{0}^{1}\left(\int_{s}^{1} p(s) p^{\prime \prime}(s) p(\xi) d \xi\right) d s-\int_{0}^{1}\left(\int_{0}^{s} p(s) p(\xi) p^{\prime \prime}(\xi) d \xi\right) d s .
\end{aligned}
$$

By interchanging the order of integration and the variables in the first integral it is easily shown that $G=0$.

Since $A+B x^{2}>0$, Eq. (11) is equivalent to the planar autonomous system

$$
\left.\begin{array}{l}
\dot{x}=y, \\
\dot{y}=\frac{-a x y^{2}-b x-c x^{3}}{A+B x^{2}} .
\end{array}\right\}
$$

First, we determine the equilibria of system (13). These are obviously given by the conditions $y=0$ and $x=0$ or

$$
x^{2}=\frac{b}{-c}=\frac{35640+495(1-9 \alpha) \beta \Omega^{2}}{2358 \beta \Omega^{2}-58320} .
$$

We may plot the sign variation of $b$ and $-c$ in the $\left(\alpha, \beta \Omega^{2}\right)$-plane. Since the equations $b=0, c=0$ are easily solved by

$$
\begin{aligned}
& b=0 \Leftrightarrow \beta \Omega^{2}=\frac{72}{9 \alpha-1} \quad\left(\alpha>\frac{1}{9}\right), \\
& c=0 \Leftrightarrow \beta \Omega^{2}=\frac{3240}{131} \simeq 24.73,
\end{aligned}
$$

we have the picture as shown in Fig. 2 (see p. 706).

Even though the model is nonlinear, it is only valid in a neighbourhood of the zero equilibrium. We thus merely investigate bifurcations from the trivial equilibrium. 


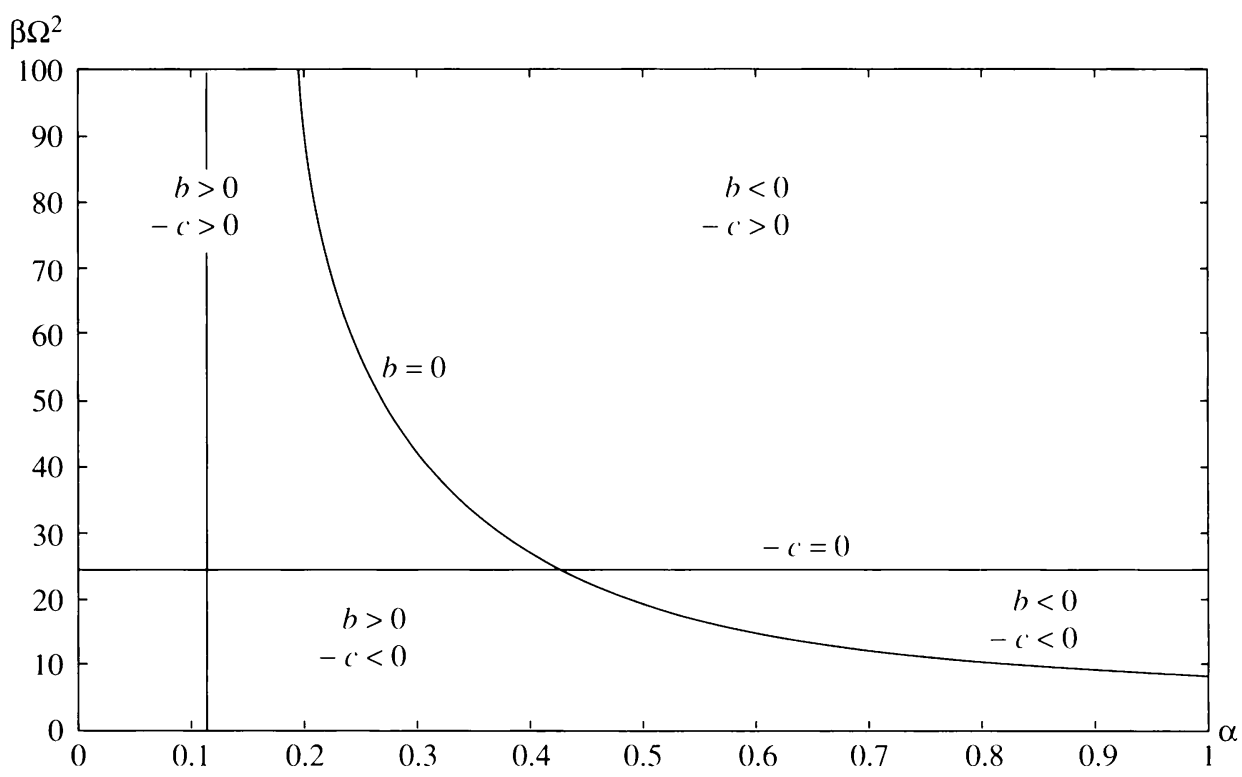

Fig. 2. The sign variation of $b$ and $-c$ from Eqs. (15) and (16) in the $\left(\alpha, \beta \Omega^{2}\right)$-plane.

For a fixed $\alpha$, we consider the behaviour as $\Omega$ increases, i.e., we move upwards along vertical lines in the $\left(\alpha, \beta \Omega^{2}\right)$-plane.

Finally, we denote that the intersection point between the curves $b=0$ and $-c=0$ corresponds to $\alpha=\frac{176}{405} \simeq 0.4346$.

This leads to the following bifurcation analysis:

CASE 1. $\alpha<\frac{1}{9}$, corresponding to $L>9 R$. Since $b$ never vanishes, no equilibria bifurcate from the trivial equilibrium.

CASE 2. $\frac{1}{9}<\alpha<\frac{176}{405}$, corresponding to $2.3 R<L<9 R$. Here $b$ vanishes in the region of positive $-c$. Hence small equilibria to Eq. (14) bifurcate from the trivial equilibrium at the point given by the formula (15) and exist for $\beta \Omega^{2}<72 /(9 \alpha-1)$.

CASE 3. $\frac{176}{405}<\alpha$, corresponding to $L<2.3 R$. Here $b$ vanishes in the region of negative $-c$. Again small equilibria to Eq. (14) bifurcate from the trivial equilibrium at the point given by expression (15) but now exist for $\beta \Omega^{2}>72 /(9 \alpha-1)$.

To proceed with the stability analysis, we investigate the eigenvalues of the Jacobian in the equilibrium $\left(x_{0}, 0\right)$. Since the system (13) is conservative (see Appendix), these eigenvalues are purely imaginary if the determinant $\Delta\left(x_{0}\right)$ of the Jacobian satisfies $\Delta\left(x_{0}\right)>0$, implying $\left(x_{0}, 0\right)$ is a center. If $\Delta\left(x_{0}\right)<0$, the eigenvalues are real and of opposite sign, so that $\left(x_{0}, 0\right)$ is a saddle point and thus unstable.

Since (13) implies the Jacobian

it is easily seen that

$$
J\left(x_{0}, 0\right)=\left[\begin{array}{cc}
\frac{b+3 c x_{0}^{2}}{A+B x_{0}^{2}}+\frac{0}{\left(b x_{0}+c x_{0}^{3}\right) \cdot 2 B x_{0}} & 1 \\
\left(A+B x_{0}^{2}\right)^{2} & 0
\end{array}\right],
$$

$$
\Delta(0)=\frac{b}{2860 \beta}
$$




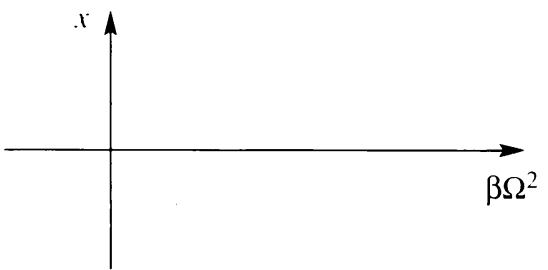

Case 1

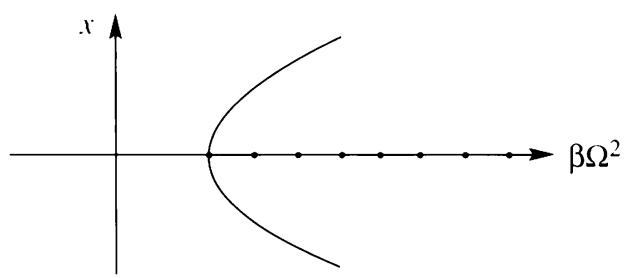

Case 3

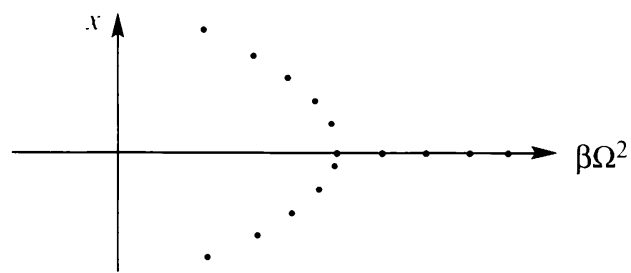

Case 2

FIG. 3. The bifurcation diagrams corresponding to the three cases. A solid curve represents a stable equilibrium, a dashed curve an unstable equilibrium. The bifurcation point is given by Eq. (15).

so the trivial equilibrium is stable for $b>0$ and unstable for $b<0$; cf. Fig. 2. If $x_{0}$ satisfies equation (14), we may after a few calculations write

$$
\Delta\left(x_{0}\right)=-\frac{b}{1430 \beta+10059 \beta x_{0}^{2}} .
$$

The denominator is positive, and hence $\left(x_{0}, 0\right)$ is stable for $b<0$ and unstable for $b>0$, which is just the opposite to the case of the trivial equilibrium.

Figure 3 shows the bifurcation diagrams and Figure 4 (see p. 708) shows the associated phase portraits. We now interpret these results in terms of the dynamical behaviour of the rod.

CASE 1. $\alpha<\frac{1}{9}$. The trivial equilibrium is always stable, and the only possible oscillations surround this equilibrium.

CASE 2. $\frac{1}{9}<\alpha<\frac{176}{405}$. As $\beta \Omega^{2}$ increases beyond $72 /(9 \alpha-1)$, the trivial equilibrium loses stability. Apparently no stable equilibria are obtained. This is referred to as a catastrophic bifurcation. However, this does not imply a breakdown of the system. Including terms of order $\leq 5$ in the model (6) and rederiving the reduced equation, one may show that the bifurcating branches in Fig. 3, Case 2 "turn around" for

$$
\beta \Omega^{2}=\frac{90.05-6.288(9 \alpha-1)+\sqrt{39.539(9 \alpha-1)^{2}+1026.3(9 \alpha-1)+8015.9}}{2.5778(9 \alpha-1)-0.1112}
$$

and change stability, such that stable equilibria exist for $\beta \Omega^{2}>72 /(9 \alpha-1)$. Since the details are rather technical, they are omitted in this paper.

CASE 3. $\frac{176}{405}<\alpha$. As $\beta \Omega^{2}$ increases beyond $72 /(9 \alpha-1)$, the trivial equilibrium loses stability, and two (symmetric) stable buckled equilibria occur. Around these, 


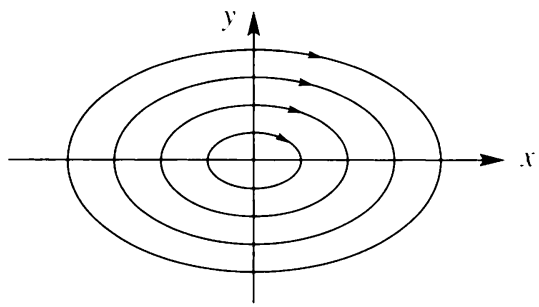

(a)

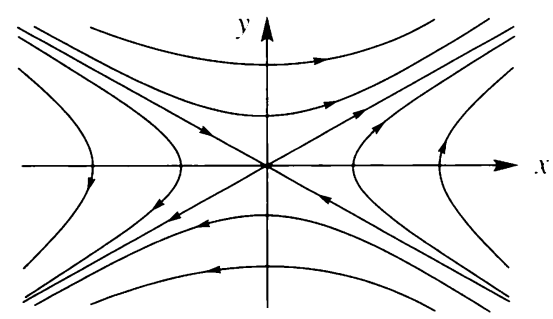

(c)

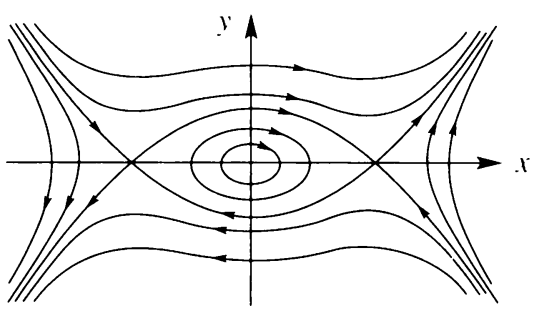

(b)

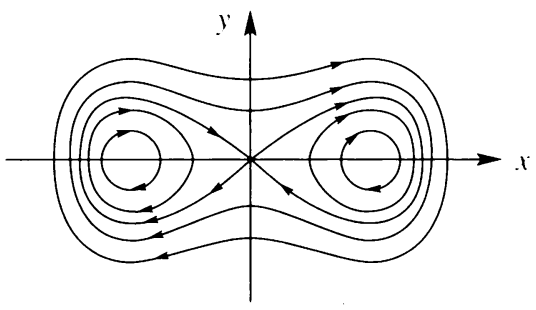

(d)

FIG. 4. Possible phase portraits for system (13). Related to Fig. 3, (a) corresponds to Case 1 and to Case 3 for $\beta \Omega^{2}<72 /(9 \alpha-1)$, (b) to Case 2 for $\beta \Omega^{2}<72 /(9 \alpha-1)$, (c) to Case 2 for $\beta \Omega^{2}>72 /(9 \alpha-1)$, and (d) to Case 3 for $\beta \Omega^{2}>72 /(9 \alpha-1)$.

small oscillations are possible. This configuration of equilibria in a conservative system implies that all trajectories outside the homoclinic orbits are closed like in Fig. 4(d), such that a stable oscillation around all three equilibrium points is possible too. Thus, in this case two types of oscillations may occur.

A linear model of the rod can only be applied to investigate the trivial equilibrium, and our results here agree perfectly with those of Mostaghel and Tadjbakhsh [1], Rammerstorfer [2], Lakin and Nachman [5], and Gürgöze [7]. This is not surprising, since the linear terms in our Eq. (6) are the same as in the above-mentioned investigations.

4. Comments on the analysis techniques. The purpose of this section is to discuss Ritz-Galerkin assumptions different from the functions (10) and other analytical methods to investigate Eq. (6). Such a discussion is useful, since the above investigation is based on approximations, the influence of which are rather unpredictable.

Apparently, the Ritz-Galerkin assumption (10) is based only on the boundary conditions but not on the differential equation. Mostaghel and Tadjbakhsh [1] propose an improved polynomial to approximate the shape of the beam under buckling, namely,

$$
\begin{aligned}
P_{2}(s)=\beta \Omega^{2} & \left(\frac{3 \alpha}{5} s^{2}-\frac{1}{5} s^{3}+\frac{1-2 \alpha}{4} s^{4}+\frac{4 \alpha-1}{10} s^{5}\right. \\
& \left.-\frac{1+8 \alpha}{60} s^{6}+\frac{35+28 \alpha}{1470} s^{7}-\frac{3}{560} s^{8}\right) .
\end{aligned}
$$


Thus, it seems natural to make the Ritz-Galerkin assumption

$$
f(s, t)=x(t) P_{2}(s)
$$

instead of (10). We have performed the calculations in REDUCE and obtained a differential equation of the same type as Eq. (11) but with other values of $A, B, a$, $b$, and $c$. It turns out that if $\alpha>0.4$, the results are very similar, but if $\alpha<0.4$, some disagreement is observed. This is due to the fact that $P_{2}$, which depends on $\alpha$, is quite similar to $P_{1}$ for $\alpha>0.4$ but has for $\alpha<0.4$ a different and rather peculiar shape compared to the first mode, which is the subject of the present investigations.

Another reasonable approach would be to replace $P_{1}$ with the exact first buckling mode in the case $\Omega=0$. This can be determined explicitly (see Collatz [14]) as

$$
P_{3}(s)=\cosh (k s)-\cos (k s)-\kappa(\sinh (k s)-\sin (k s)),
$$

where $k=1.8751$ and $\kappa=0.7341$. But for $s \in[0,1]$, this function (21) is very close to $P_{1}(s)$, so that the results of the integrations in the Galerkin procedure are very similar to those in Sec. 3 .

Another way to determine approximate solutions to a nonlinear problem is to apply perturbation techniques, such as the Poincaré-Lindstedt series; see, e.g., Iooss and Joseph [15]. But in order to apply this method, the explicit solution to the linear equation (7) has to be known, and that is not the case here.

However, one could solve (7) by means of a power series in $s$. But this method has two disadvantages; namely, (i): The recursion formula depends on $\alpha$ and $\beta \Omega^{2}$, such that the coefficients in the power series become very complicated expressions in these parameters, and (ii): The boundary conditions in $s=1$ are difficult to convert to conditions on the coefficients. Our preliminary attempts in this direction discourage further efforts.

5. Conclusions. We have proposed and investigated a nonlinear model for a beam of length $L$ clamped radially to the interior of a rotating ring with radius $R$. The terms in the model Eq. (6) arising from the Coriolis forces have no influence on the dynamics predicted by the investigations. By Galerkin's method, the model equation is reduced to an ODE, and a phase plane analysis shows the following bifurcation and stability behaviour:

CASE 1. If $L>9 R$, no small equilibria bifurcate from the trivial equilibrium, and the trivial equilibrium is always stable. Thus, independent of the angular velocity $\Omega$, no buckling occurs, and the beam will oscillate around the trivial equilibrium. This is in agreement with earlier investigations of linear models.

CASE 2. If $2.3 R<L<9 R$, the trivial solution loses stability at $\beta \Omega^{2}=$ $72 /(9 R / L-1)$. This value is in agreement with the linear theory. Small buckled equilibria bifurcate from the trivial solution at this point, but they exist for $\beta \Omega^{2}<72 /(9 R / L-1)$ and are unstable. Thus apparently, no stable equilibria exist for $\beta \Omega^{2}>72 /(9 R / L-1)$. However, including terms of fifth degree in the model confirm that the branches of unstable equilibria "turn around" and change stability through another bifurcation. Detailed investigation of this phenomenon requires unfolding of the singularity occurring when $\frac{R}{L}=\frac{176}{405}$ and is omitted here. 
CASE 3. If $L<2.3 R$, the trivial equilibrium loses stability at $\beta \Omega^{2}=$ $72 /(9 R / L-1)$, and small buckled equilibria bifurcate from the trivial equilibrium just as in Case 2. But here the bifurcating equilibria exist for $\beta \Omega^{2}>72 /(9 R / L-1)$ and are stable, and therefore two types of oscillations of the beam are possible: Either small oscillations around a buckled equilibrium or larger oscillations around the trivial equilibrium, surrounding all equilibria.

In general, we may then conclude that the greater the length of the beam relative to the ring radius, the more stable the trivial solution. Also, when the trivial equilibrium does lose stability, the beam may oscillate around buckled equilibria as well as making larger oscillations around the trivial equilibrium.

Appendix. In this appendix, we briefly discuss the mathematical background for the linearizations in Sec. 3.

Consider the system of two nonlinear first-order autonomous ODEs given by

$$
\dot{x}=P(x, y), \quad \dot{y}=Q(x, y) .
$$

A nonconstant function $V(x, y)$ is called an integral of the system (A1), if $\dot{V}=0$ along the trajectories of $(\mathrm{A} 1)$, i.e.,

$$
V_{x}(x, y) P(x, y)+V_{y}(x, y) Q(x, y)=0 .
$$

The system (A1) is called conservative if it has an integral defined in the entire phase plane.

The trajectories of a conservative system must therefore lie on the level curves of the integral.

Partial differentiation of equation (A2) shows that if $\left(x_{0}, y_{0}\right)$ is an equilibrium of (A1) with $\operatorname{det} J\left(x_{0}, y_{0}\right) \neq 0$ and $V$ is an integral of (A1), then $V_{x}\left(x_{0}, y_{0}\right)=$ $V_{y}\left(x_{0}, y_{0}\right)=0$. Thus the graph of $V$ has a horizontal tangent plane in $\left(x_{0}, y_{0}\right)$. If, furthermore, $V$ has an extremum in $\left(x_{0}, y_{0}\right)$, then the level curves of $V$ are closed in a neighbourhood of $\left(x_{0}, y_{0}\right)$, and thus the equilibrium $\left(x_{0}, y_{0}\right)$ is a center.

Further partial differentiations of equation (A2) and some niggling work show that if $\operatorname{det} J\left(x_{0}, y_{0}\right)>0$ and $V_{y y}\left(x_{0}, y_{0}\right) \neq 0$, then $V$ has an extremum in $\left(x_{0}, y_{0}\right)$. Thus if $\left(x_{0}, y_{0}\right)$ is an equilibrium of the system (A1), det $J\left(x_{0}, y_{0}\right)>0$ and $V_{y y}\left(x_{0}, y_{0}\right) \neq 0$, then $\left(x_{0}, y_{0}\right)$ is a center.

For the concrete system (13), it is not hard to verify that the function

$$
V(x, y)=y^{2}\left(A+B x^{2}\right)^{a / B}+\int_{0}^{x}\left(A+B u^{2}\right)^{a / B-1}\left(2 b u-2 c u^{3}\right) d u
$$

is an integral, such that (13) is conservative. Furthermore,

$$
V_{y y}(x, y)=2\left(A+B x^{2}\right)^{a / B}>0,
$$

and hence any equilibrium $\left(x_{0}, 0\right)$ of the system (13) with $\operatorname{det} J\left(x_{0}, 0\right)>0$ is a center. If det $J\left(x_{0}, 0\right)<0, J\left(x_{0}, 0\right)$ has two real eigenvalues of opposite sign, and $\left(x_{0}, 0\right)$ is a saddle point. Thus in system (13), the sign of $\operatorname{det} J\left(x_{0}, 0\right)$ determines the stability of $\left(x_{0}, 0\right)$ completely, so that the linearizations are valid. 


\section{REFERENCES}

[1] N. Mostaghel and I. Tadjbakhsh, Buckling of rotating rods and plates, Internat. J. Mech. Sci. 15, 429-434 (1973)

[2] F. G. Rammerstorfer, Comment on buckling of rotating rods and plates, Internat. J. Mech. Sci. 16, 515-517 (1974)

[3] A. Nachman, The buckling of rotating rods, J. Appl. Mech. 42, 222-224 (1975)

[4] J. T. S. Wang, On the buckling of rotating rods, Internat. J. Mech. Sci. 18, 407-411 (1976)

[5] W. D. Lakin and A. Nachman, Unstable vibrations and buckling of rotating flexible rods, Quart. Appl. Math. 35, 479-493 (1978)

[6] D. A. Peters and D. H. Hodges, In-plane vibration and buckling of a rotating beam clamped off the axis of rotation, J. Appl. Mech. 47, 398-402 (1980)

[7] M. Gürgöze, On the dynamical behaviour of a rotating beam, J. Sound and Vibration 143, 356-363 (1990)

[8] H. I. Weber, A note on the stability of a rod subjected to compression by centrifugal force, J. Sound and Vibration 46, 105-111 (1976)

[9] A. G. Hernried and G. B. Gustafson, On the dynamic response of a single-degree-of-freedom structure attached to the interior of a rotating rigid ring, J. Appl. Mech. 55, 201-205 (1988)

[10] D. A. Peters and D. H. Hodges, Discussion on the dynamic response of a single-degree-of-freedom structure attached to the interior of a rotating rigid ring, J. Appl. Mech. 55, 747-748 (1988)

[11] W. Kliem, Good and bad mathematical modelling in mechanics, Teaching of Mathematical Modelling and Applications, M. Niss, W. Blum, and I. Huntley (Eds.), Ellis Horwood, Chichester, 1991, pp. 384-392

[12] A. C. Hearn, Reduce, User's Manual, Version 3.3, The Rand Corporation, Santa Monica, CA, Rand Publication CP 78 (Rev. 7/87), 1987

[13] G. Rayna, Reduce: Software for Algebraic Computation, Springer-Verlag, New York, 1987

[14] L. Collatz, Eigenwertaufgaben mit technischen Anwendungen, Akademische Verlagsgesellschaft, Leipzig, 1963

[15] G. Iooss and D. D. Joseph, Elementary Stability and Bifurcation Theory, Springer-Verlag, New York, 1980

[16] E. A. Coddington and N. Levinson, Theory of Ordinary Differential Equations, McGraw-Hill, New York, 1955 